\title{
Re-treatment of Maxillary Premolar with Calcified Canals and Inadequate Obturation
}

\author{
Viresh Chopra $^{1 *}$, Harneet Chopra ${ }^{2}$ and Gurdeep Singh ${ }^{2}$ \\ ${ }^{1}$ Course Leader, Endodontology, Senior Lecturer, Adult Restorative Dentistry, Oman \\ Dental College, Muscat, Oman \\ ${ }^{2}$ Senior Lecturer, Adult Restorative Dentistry, Oman Dental College, Muscat, Oman \\ *Corresponding Author: Viresh Chopra, Course Leader, Endodontology, Senior \\ Lecturer, Adult Restorative Dentistry, Oman Dental College, Muscat, Oman.
}

Received: July 13, 2021

Published: November 01, 2021

(C) All rights are reserved by Viresh Chopra., et al.

\begin{abstract}
Treatment/Retreatment in endodontics has changed fundamentally in the last few years following the introduction of the surgical operating microscope. Cases that once seemed impossible became easy and exciting to operate. Although initial root canal therapy has been shown to be a predictable procedure with a high degree of success, failures can occur after treatment. As fewer teeth are extracted, an increasing number of retreatment cases have been recorded in the past 15 years. Utilization of the enhanced magnification devices in endodontic retreatment has brought significant advantages and has expanded the scope of indications using a non-surgical approach. Procedures such as bypassing a ledge, removing broken instrument or repair a perforation have become considerably more reliable.

This case report presents a case of failed root canal emphasising on the various factors such as short root canal obturation, inadequate cleaning and shaping and calcified canals leading to failure of the treatment. It also lays down the protocol to manage calcified canals, disinfect the root canal system in an effective manner so as to induce healing from the tissues.
\end{abstract}

Keywords: Maxillary Premolar; Calcified Canals; Obturation

\section{Introduction}

Success in root canal treatment is a result of thorough cleaning and shaping, disinfection and adequate three-dimensional obturation. Compromising any of the above steps can lead to failure of the root canal therapy. Reading a preoperative periapical radiograph is of prime steps that needs to be done carefully. Though, they are 2 dimensional but they do provide us with an approximate type and number of root canals. Apical calcification of the canals can result from multiple factors and can pose a significant challenge in cleaning and shaping of the root canal. Failure to clean the calcified part of the root canal might result in inadequate cleaning and shaping resulting in persistence of the diseased tissue.
This case report aims to present a Case of Symptomatic Apical Periodontitis (SAP) with chronic abscess associated with a previous root canal treatment with inadequate obturation in a maxillary premolar.

\section{Description of the Case}

\section{Patient information}

A male patient aged 29 years with non-contributory medical history reported to the clinic with chief complaint of pain on biting or pressure in upper right posterior region of the oral cavity. There is a history of root canal therapy in maxillary right second premolar. 


\section{Clinical examination findings}

Tenderness to percussion present in tooth \#15. Buccal vestibule had a slight intraoral swelling, which was tender to touch.

\section{Radiographic findings}

Previous root canal treatment in 15 with short obturation is visible on periapical radiograph. The root is associated with periapical radiolucency. The apical part of the canals suspected to be calcified (which are Unobturated) (Figure 1).

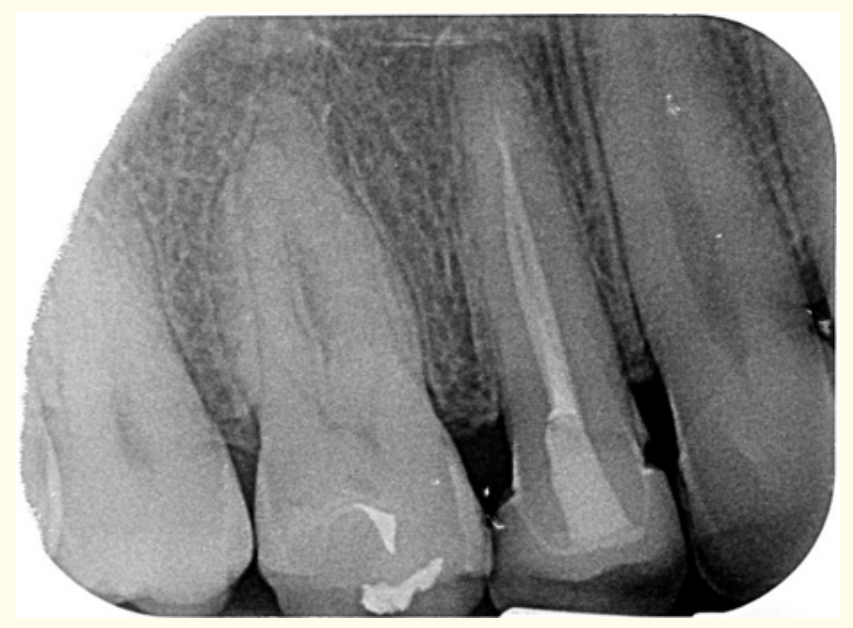

Figure 1: Periapical radiograph inadequate root canal treatment in 15. Periapical radiolucency associated with the root of 15 can also be seen.

\section{Treatment plan}

The following choices were discussed with the patient regarding the root canal retreatment:

1. Re-rct through the present all ceramic crown.

2. Re-rct after removal of the all ceramic crown.

Patient chose option 1. However, it was made clear that all ceramic crown can decement under the clamp load or vibrations from the high/low speed machines. In that case it needs to be replaced. Patient agreed and we started the re-rct through the present crown.
Step wise procedures in the first visit:

1. Buccal infiltration anesthesia was administered, the tooth was isolated with rubber dam (Figure 2).

2. Endodontic access was gained through the crown and previous gutta perchas were found (Figure 3).

3. Gutta perchas were removed with the help of Endoshaper file from FKG used at 2500rpm (Figure 4) (Video 1). Intracanal medicament was placed and patient recalled for second appointment (Figure 5).

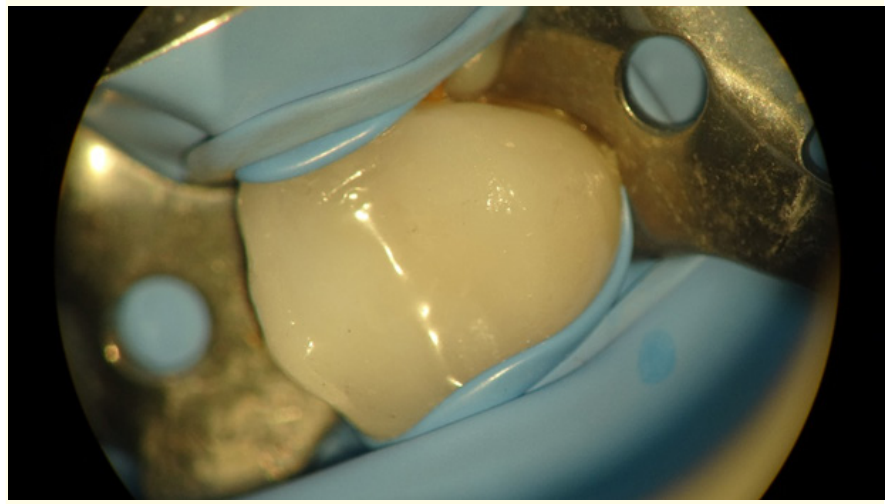

Figure 2: Tooth 15 under isolation with all ceramic crown before starting with the retreatment procedure.

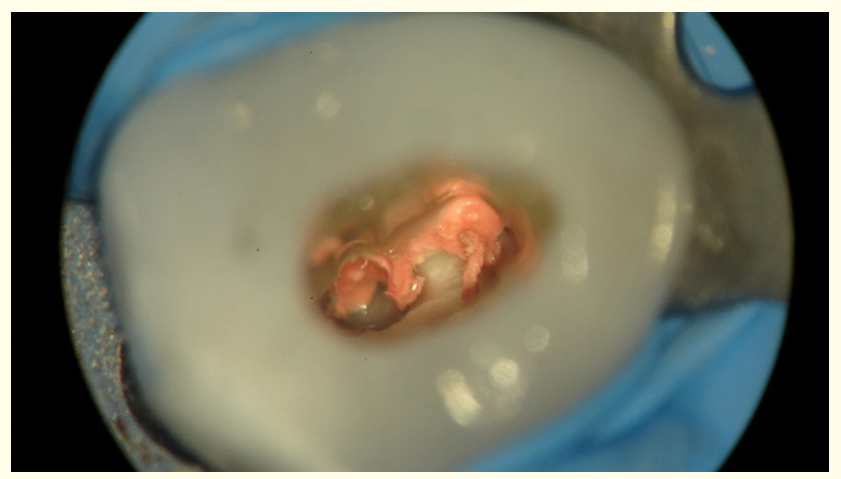

Figure 3: Endodontic access cavity made through the ceramic crown. Older gutta perchas located. 


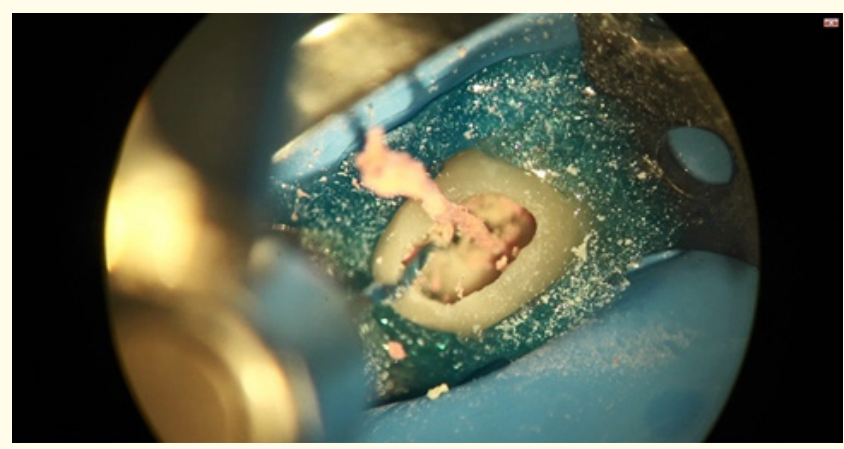

Figure 4: Removal of gutta perchas with Endoshaper files from FKG.

video 2: rewmoval of GP with rotary file. MP4 https://actascientific.com/ASDS/video/Case $\% 2018 \% 20$ video\%201\%20REMOVING\%20GP\%20WITH\%20ULTRASONIC. MP4

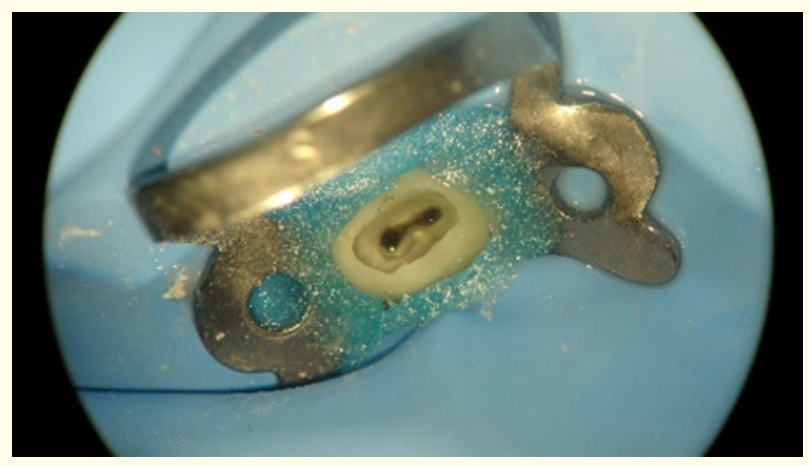

Figure 5: Previous gutta percha removed from the root canals.

Treatment procedure for the second appointment

1. The all-ceramic crown came out during clamp placement. Sodium hypochlorite was used as an endodontic irrigant. $08 \mathrm{~K}$ and $10 \mathrm{~K}$ endodontic files were pre curved and used to open the calcified canals. C+ files were also used in order to open up the apically calcified part of the root canals. These files were used with passive motion in presence of the irrigants. After negotiation of the apical part, working length was determined with Electronic apex locator and verified with a periapical radiograph (Figure 6).
2. Both the canals were finally prepared up to 25/04 (Figure 7).

3. Master cone fit was verified clinically with tug back. Also, periapical radiograph was taken to verify their fit at the correct working length (Figure 8).

4. Obturation was done using GP and AH Plus as a root canal sealer. Warm vertical condensation technique was used for obturation (Figure 9).

5. Final obturation was verified with an immediate postoperative radiograph (Figure 10).

6. 6-month recall of the case showed adequate healing and patient remained asymptomatic (Figure 11).

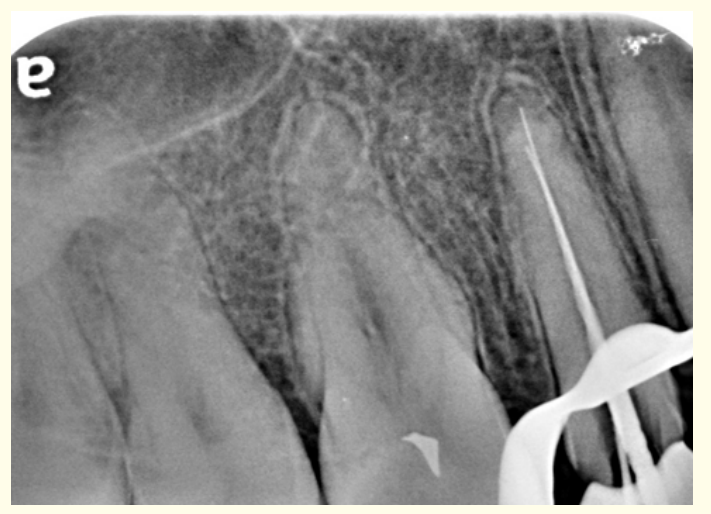

Figure 6: Periapical radiograph verifying the working length.

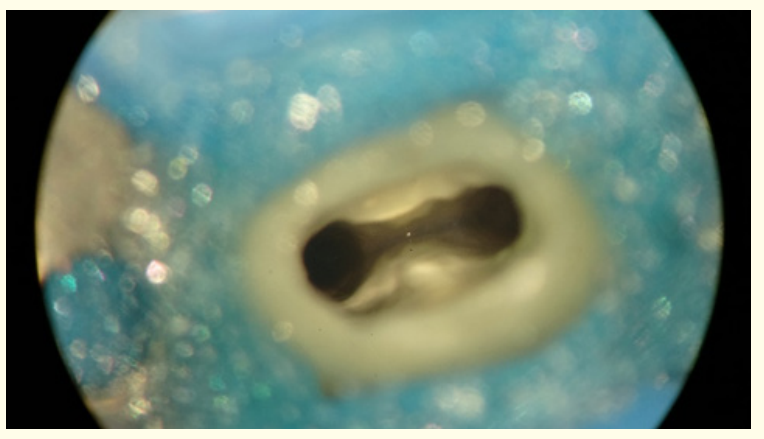

Figure 7: Canals prepared up-to 25/04.

Citation: Viresh Chopra., et al. "Re-treatment of Maxillary Premolar with Calcified Canals and Inadequate Obturation". Acta Scientific Dental Sciences 5.12 (2021): 02-07. 


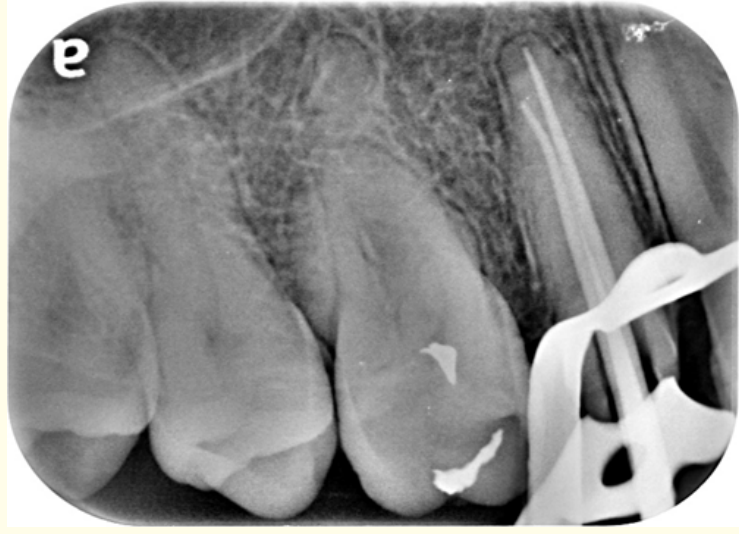

Figure 8: Periapical radiograph verifying the master cones for obturation.

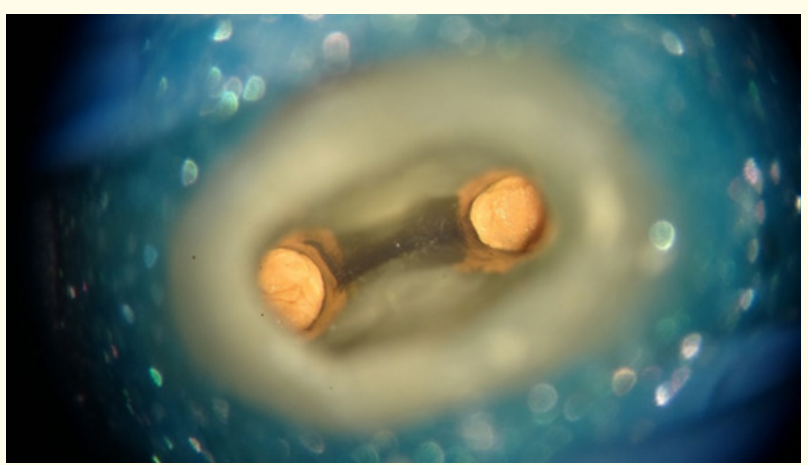

Figure 9: Obturation of the root canal system.

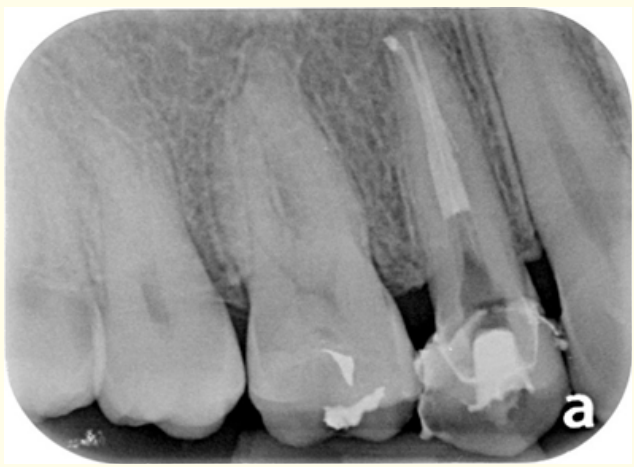

Figure 10: Immediate postoperative radiograph to verify the obturation.

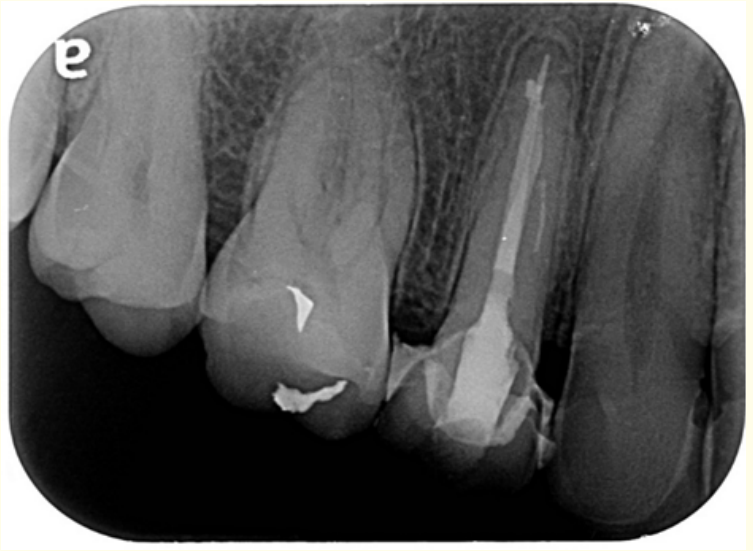

Figure 11: 6-month recall radiograph showing intact crown, obturation and ongoing healing of the periapical area.

\section{Discussion}

An endodontically treated tooth needs to be evaluated clinically and radiographically during follow ups for its root canal treatment to be called successful. Multiple factors are listed to cause failure of the initial endodontic treatment [1]:

1. Persistence of bacteria (intra-canal and extra-canal)

2. Inadequate quality of obturation (canals that are poorly cleaned and obturated)

3. Overextended of root filling materials

4. Improper coronal seal (leakage)

5. Untreated canals (both major and accessory)

6. Iatrogenic procedural errors such as poor access cavity design

7. Complications of instrumentation (ledges, perforations, or separated instruments).

The aim of this case report is to highlight short obturation causing failure of the root canal treatment. The maxillary first premolar presents with a high percentage in variation in root anatomy and root canal morphology such as anomalies with variation, different in number and anatomy of roots. Maxillary first premolars with two canals may be considered as normal but in some cases the 2 
canals may have calcifications or curvatures which make the root canal treatment difficult [2].

With proper disinfection and debridement of canals, quality of obturation also plays an important role in success of root canal treatment. It was the most important factor in the success of the root canal treatment in a study carried out on around 1000 endodontically treated teeth [3]. In addition, Hoen., et al. showed that $65 \%$ of the cases showed poor quality obturation whereas $42 \%$ of the teeth had untreated canals [4]. Buarrieshi-Nusair, et al. and Peak., et al. they recorded more than 34\% under fillings in their studies respectively $[5,6]$.

As a result of necrosis of the dental pulp, the pulp chamber becomes progressively colonized by microorganisms from the oral cavity and interconnected systems [7]. Formation of abscesses, cysts and periapical granulomas can result from immuno-pathological mechanisms between the root apex and the periapical tissue [8].

In the present case, ineffective cleaning and shaping of the apical part of the root canal lead to short obturation with inadequate apical seal. This gave a chance to the microorganisms to proliferate and start the immune-pathological mechanisms resulting in apical periodontitis.

Treatment planning is a very important and vital part for the successful execution of the root canal retreatment procedure. Reassessing of root canals is challenging and requires a thorough understanding of the basic root canal anatomy along with high level of proficiency and clinical skill of the dentist. Good quality radiographs and magnification tools such as loops or dental operating microscopes are essential for the detection of additional roots and root canals [9]. Also, the outline of the access cavity is decided by the size and shape of the pulp chamber and the position of the root canal orifices. Balleri., et al. in their study suggested a T shaped access outline since this can allow for straight line access to the canals [10].

Successful removal of previous gutta perchas and gaining access to the entire root canal length was vital in complete retreatment of this case. Once the gutta perchas were removed and the calcified canals were opened with $\mathrm{C}+$ files and adequate biomechanical preparation done in order to achieve a good apical seal with obturation of the root canal system.

\section{Conclusion}

Root canal system needs to be thoroughly cleaned, shaped, disinfected and obturated three dimensionally during the treatment in order to achieve success.

Failure to negotiate the whole length of the canal will lead to inadequate cleaning and shaping leaving plethora of residual microorganisms behind. It also creates a favourable environment for the residual microorganisms to proliferate and lead to periapical changes resulting in failure of the root canal treatment as seen in this case.

Therefore, the root canals should be, coronally and apically sealed, properly up to the full working length in order to achieve success in endodontic treatment.

\section{Bibliography}

1. Siqueira Jr JF. "Aetiology of root canal treatment failure: why well-treated teeth can fail". International Endodontic Journal 34.1 (2001): 1-10.

2. Atieh MA. "Root and canal morphology of maxillary first premolars in a Saudi population". The Journal of Contemporary Dental Practice 9.1 (2008): 46-53.

3. Tronstad L., et al. "Influence of coronal restorations on the periapical health of endodontically treated teeth". Dental Traumatology 16.5 (2000): 218-221.

4. Hoen MM and Pink FE. "Contemporary endodontic retreatments: an analysis based on clinical treatment findings". Journal of Endodontics 28.12 (2002): 834-836.

5. Barrieshi-Nusair K., et al. "Radiographic technical quality of root canal treatment performed by dental students at the Dental Teaching Center in Jordan". Journal of Dentistry 32.4 (2004): 301-307.

6. Peak J., et al. "The outcome of root canal treatment. A retrospective study within the armed forces (Royal Air Force)". British Dental Journal 190.3 (2001): 140-144.

7. Soares J. "Microbiota dos canais radiculares associada às lesões periapicais crônicas e sua significância clínica”. Journal Brasileiro de Endodontia 3 (2002): 106-117.

8. Soares JA and César CAS. "Avaliação clínica e radiográfica do tratamento endodôntico em sessão única de dentes com lesões periapicais crônicas". Pesquisa Odontológica Brasileira 15 (2001): 138-144. 
9. Bellizzi R and Hartwell G. "Radiographic evaluation of root canal anatomy of in vivo endodontically treated maxillary premolars". Journal of Endodontics 11.1 (1985): 37-39.

10. Balleri P., et al. "Primer premolar superior com tres raices". Endodontic Practice 3.2 (1997): 13-15.

Volume 5 Issue 12 December 2021

(C) All rights are reserved by Viresh Chopra., et al.

Citation: Viresh Chopra., et al. "Re-treatment of Maxillary Premolar with Calcified Canals and Inadequate Obturation". Acta Scientific Dental Sciences 5.12 (2021): 02-07. 